\title{
A cluster-randomized controlled trial evaluating the effect of culturally-appropriate hypertension education among Afro-Surinamese and Ghanaian patients in Dutch general practice: study protocol
}

\author{
Joke A Haafkens*1, Erik JAJ Beune ${ }^{1}$, Eric P Moll van Charante ${ }^{1}$ and \\ Charles O Agyemang 2
} \begin{abstract}
Medicine, Academic Medical Center, University of Amsterdam, The Netherlands
Email: Joke A Haafkens* - j.a.haafkens@amc.uva.nl; Erik JAJ Beune - e.j.beune@amc.uva.nl; Eric P Moll van

Charante - e.p.mollvancharante@amc.uva.nl; Charles O Agyemang - c.o.ayyemang@amc.uva.nl

* Corresponding author
\end{abstract}

Address: ${ }^{1}$ Department of General Practice, Amsterdam Medical Center, University of Amsterdam, The Netherlands and ${ }^{2}$ Department of Social

Published: 22 October 2009

BMC Health Services Research 2009, 9:193 doi:10.1186/1472-6963-9-193

This article is available from: http://www.biomedcentral.com/1472-6963/9/193

(c) 2009 Haafkens et al; licensee BioMed Central Ltd.

This is an Open Access article distributed under the terms of the Creative Commons Attribution License (http://creativecommons.org/licenses/by/2.0), which permits unrestricted use, distribution, and reproduction in any medium, provided the original work is properly cited.
Received: 7 September 2009

Accepted: 22 October 2009

which permits unrestricted use, distribution, and reproduction in any medium, provided the original work is properly cited.

\begin{abstract}
Background: Individuals of African descent living in western countries have increased rates of hypertension and hypertension-related complications. Poor adherence to hypertension treatment (medication and lifestyle changes) has been identified as one of the most important modifiable causes for the observed disparities in hypertension related complications, with patient education being recommended to improve adherence. Despite evidence that culturallyappropriate patient education may improve the overall quality of care for ethnic minority patients, few studies have focused on how hypertensive individuals of African descent respond to this approach. This paper describes the design of a study that compares the effectiveness of culturally-appropriate hypertension education with that of the standard approach among Surinamese and Ghanaian hypertensive patients with an elevated blood pressure in Dutch primary care practices.
\end{abstract}

Methods/Design: A cluster-randomized controlled trial will be conducted in four primary care practices in Amsterdam, all offering hypertension care according to Dutch clinical guidelines. After randomization, patients in the usual care sites $(n=2)$ will receive standard hypertension education. Patients in the intervention sites $(n=2)$ will receive three culturallyappropriate hypertension education sessions, culturally-specific educational materials and targeted lifestyle support. The primary outcome will be the proportion of patients with a reduction in systolic blood pressure $\geq 10 \mathrm{mmHg}$ at eight months after the start of the trial. The secondary outcomes will be the proportion of patients with self-reported adherence to (i) medication and (ii) lifestyle recommendations at eight months after the start of the trial. The study will enrol 148 patients (74 per condition, 37 per site). Eligibility criteria for patients of either sex will be: current diagnosis of hypertension, self-identified Afro-Surinamese or Ghanaian, $\geq 20$ years, and baseline blood pressure $\geq 140 / 90 \mathrm{mmHg}$. Primary and secondary outcomes will be measured at baseline and at $3 \mathrm{I} / 2,6 \mathrm{I} / 2$, and eight months. Other measurements will be performed at baseline and eight months.

Discussion: The findings will provide new knowledge on how to improve blood pressure control and patient adherence in ethnic minority persons with a high risk of negative hypertension-related health outcomes.

Trial registration: ISRCTN35675524 


\section{Background}

Hypertension (HTN) is a major risk factor for cardiovascular morbidity and mortality [1]. In western countries, ethnic minority populations of African descent are disproportionately affected by HTN and HTN-related cardiovascular morbidity and mortality [2-4]. This has also been observed among two major immigrant groups of African descent in the Netherlands: African-Surinamese from the former Dutch colony of Suriname (hereafter referred to as Surinamese) [5], and Ghanaians [6,7]. Poor adherence to prescribed medication and lifestyle changes such as weight control, regular physical activity, avoidance of tobacco, and a moderate intake of salt and alcohol, has been identified as one of the most important modifiable causes for the observed disparities in hypertension related complications [8,9]. Enhancing patient adherence to such therapeutic measures can be an important first step towards eliminating disparities [4,6,9-11].

In the Netherlands, general practitioners (GPs) play an important role in the treatment of HTN. European and Dutch primary care guidelines recommend patient education as a means of improving patients' motivation for and ability to adhere to HTN treatment goals [12,13]. Medical perceptions of disease and treatment can differ from lay perceptions [14], and patients can have a major impact on adherence to treatment [15-17]. HTN educators are therefore advised to employ "patient-centred" educational approaches that allow them to explore individual patients' beliefs and needs, and to find common ground regarding treatment [18]. The Dutch guidelines recommend focusing on the "5 As" (ask, assess, advise, assist and arrange) [19]. Studies from the UK and the USA have shown that patient beliefs about HTN and treatment can differ between ethnic groups [20-24]. This was also a finding from one of our previous studies, which focused on White Dutch, Surinamese and Ghanaian hypertensive patients living in the Netherlands [25]. Indeed, there is increasing evidence, mainly from diabetes research, that "culturally sensitive" patient education may have a positive influence on medication use and lifestyle changes among ethnic minority patients [26,27]. Culturallyappropriate patient education typically combines the principle of "patient-centred" care with that of "culturally competent" care [18]. To date, however, the European and Dutch HTN guidelines for GPs have not provided any recommendations on how HTN educators might address cultural variations in patients' perceptions of HTN. Moreover, the literature offers few descriptions or evaluations of means by which this might be achieved $[11,28]$.

In a previous project, "Under Pressure 1", we developed a multi-component provider intervention to facilitate culturally-appropriate HTN education (CAHE) in Dutch primary care practices. This intervention mainly consisted of a CAHE toolkit that could be used to supplement the standard approach to HTN education recommended by Dutch clinical guidelines [1]. To support HTN educators using the toolkit, we developed an educational course and a framework for feedback meetings. A pilot undertaken in six primary care health centres (PCHC) in South East Amsterdam demonstrated that healthcare providers who had received the intervention thought it more important to take a patient's cultural background into account when delivering care. They also experienced fewer barriers to delivering culturally-appropriate health education to patients with cardiovascular problems than the control group (Beune EJAJ, Haafkens JA, Mohrs J, Stronks K, Bindels PJE: Pilot study evaluating the effects of an intervention to enhance culturally appropriate hypertension education among health care providers in a primary care setting, submitted). In the pilot practices, general practice assistants (GP assistants) and nurse practitioners (NP) were responsible for conducting HTN education under the supervision of GPs. A qualitative study of the experiences of the professionals who had piloted the intervention revealed a number of contextual barriers that had hampered the implementation of the CAHE toolkit in daily practice (Beune EJAJ, Haafkens JA, Bindels PJE: Pilot study of barriers and enablers influencing the implementation of an intervention to stimulate culturally appropriate hypertension education in a primary care setting, submitted). Frequently-mentioned problems included PCHCs being under-resourced, ongoing organizational changes, limited access to office space, the rapid turnover of GP assistants, and the fact that initially, GP assistants lacked the skills to conduct patient-centred HTN. Fortunately, many of these barriers could be addressed using well-known strategies for supporting innovations in primary care [29-31]. We therefore concluded that it would be feasible for Dutch primary care practices to adopt and implement the intervention.

In this paper, we describe the design of and rationale for our next project, "Under Pressure 2 (UP2)". The aim of UP2 is to test, using a cluster-randomized trial, the effectiveness of the previously-developed CAHE intervention among hypertensive Surinamese and Ghanaian patients. The latter should be receiving care in a Dutch primary care setting, and should have an elevated blood pressure (that is, greater than or equal to $140 / 90 \mathrm{mmHg}$ ). Our primary hypothesis is that a higher proportion of patients randomized to the CAHE intervention will have a reduction in systolic blood pressure (SBP) greater than or equal to 10 $\mathrm{mmHg}$ at eight months after the start of the intervention, as compared to those randomized to the usual care condition. Our secondary hypotheses are that a higher proportion of patients randomized to the intervention will be adherent to the prescribed (i) medication and (ii) lifestyle recommendations at eight months after the start of the 
intervention, as compared to those randomized to the usual care condition.

\section{Methods}

\section{Study design}

The study will consist of a cluster-randomized controlled trial with two conditions: an intervention condition (IC) and a usual care condition (UC), as shown in Figure 1. Using a balanced design, four PCHCs will be randomly assigned to the IC $(n=2)$ or the UC $(n=2)$. Prior to randomization, the PCHCs will be matched on the size of the practices. A member of the Academic Medical Center's (AMC) Data Management Services team will carry out the randomization of the practices, and this team member will be blinded for the practices' identities. In those PCHCs assigned to the UC, the patients studied will receive the standard $\mathrm{HTN}$ education, based on the recommendations in the Dutch clinical guidelines [1]. In the PCHCs assigned to the IC, meanwhile, the patients studied will receive three CAHE sessions at two weeks post baseline, and three and six months thereafter. Data will be collected at baseline, during two office visits three-and-ahalf and six-and-a-half months thereafter, and at eight months past baseline. The primary and secondary outcomes will be calculated based on data collected at baseline and eight months thereafter.

\section{Study sites}

South East Amsterdam is one of the areas in the Netherlands that has a relatively high percentage of Surinamese and Ghanaian inhabitants. We have already selected four PCHCs in South East Amsterdam for the study. The inclusion criteria for each of the sites are that they (i) provide HTN care according to a practice protocol based on the Dutch clinical guidelines [1], and (ii) are not participating in similar studies to improve cardiovascular risk management.

\section{Study population}

To be recruited for the study, patients have to fulfil the following eligibility criteria: (i) self-identified as Surinamese or Ghanaian; (ii) aged 20 or over; (iii) diagnosis of HTN with the International Classification of Primary care ICPC codes K85, K86 or K87; and (iv) an uncontrolled BP at the last office visit. Following the Dutch clinical guidelines, an elevated BP is defined as SBP/diastolic blood pressure $(\mathrm{DBP}) \geq 140 / 90 \mathrm{mmHg}$. In addition, all patients must have an elevated $\mathrm{BP}$ at the baseline assessment meeting. Patients will be excluded if they have type 1 or type 2 diabetes, on the grounds that many diabetes patients with HTN may already be following diabetes education programmes that share characteristics with the intervention that will be offered in this trial. Patients will also be excluded if they are participating in other cardiovascular disease-related trials, if the GP who is treating them judges them to be unfit for participation (e.g. due to co-morbidity), or if they are unable or unwilling to provide informed consent.

\section{Sample size calculation}

Our power analysis for sample size is based on the primary outcome measure of the study: SBP at baseline minus SBP at eight months after baseline. SBP has been selected because it is the most important factor for determining a patient's cardiovascular risk profile [1]. Furthermore, in almost all cases, the DBP will become lower if the SBP becomes lower. Following the literature, we consider a reduction of the SBP of $10 \mathrm{mmHg}$ to be a clinically relevant difference [16,32].

The following rationale was used for the power calculation of the sample. The trial will be conducted in four PCHCs. We assume that patients in the IC and the UC will have similar demographic characteristics and that they will have been treated similarly, in line with the Dutch clinical guidelines [1]. We therefore expect there to be no differences between the four PCHCs with respect to the mean BP of patients with HTN. We can assume that the inter-cluster correlation is low (3\%), because this is a ratio of the between-cluster and the low intra-cluster variance. In order to demonstrate a statistical difference of 10 $\mathrm{mmHg}$ with a standard deviation (SD) of 15 and a twosided alpha of $5 \%$ and a power of $80 \%$, we need a sample size of 148 patients (74 per treatment condition and 37 per PCHC). As we expect a drop-out rate of $40 \%$ prior to inclusion, we aim to recruit 246 patients.

Registry data from the four PCHCs that have been included in the study show that each PCHC has about 100 Surinamese and 38 Ghanaian registered patients who are being treated for HTN (K85,86, K87). Based on data from Agyemang et al. [5], it is estimated that about $63 \%$ of these patients will have a blood pressure $>140 / 90 \mathrm{mmHg}$. We therefore expect that we will be able to obtain an adequate sample size for this study.

\section{Approvals and data and safety monitoring}

The study has been approved by the AMC's Medical Ethics Committee, which forms part of the University of Amsterdam in the Netherlands (protocol ID MEC09/070). Participants will provide written informed consent prior to enrolment. Recruitment procedures will be conducted in accordance with the Dutch Medical Research Involving Human Subjects Act and the World Medical Association Declaration of Helsinki.

\section{Patient recruitment}

The following steps will be taken in order to recruit patients. First, the electronic medical records (EMR) from the four selected PCHCs will be reviewed in order to select 


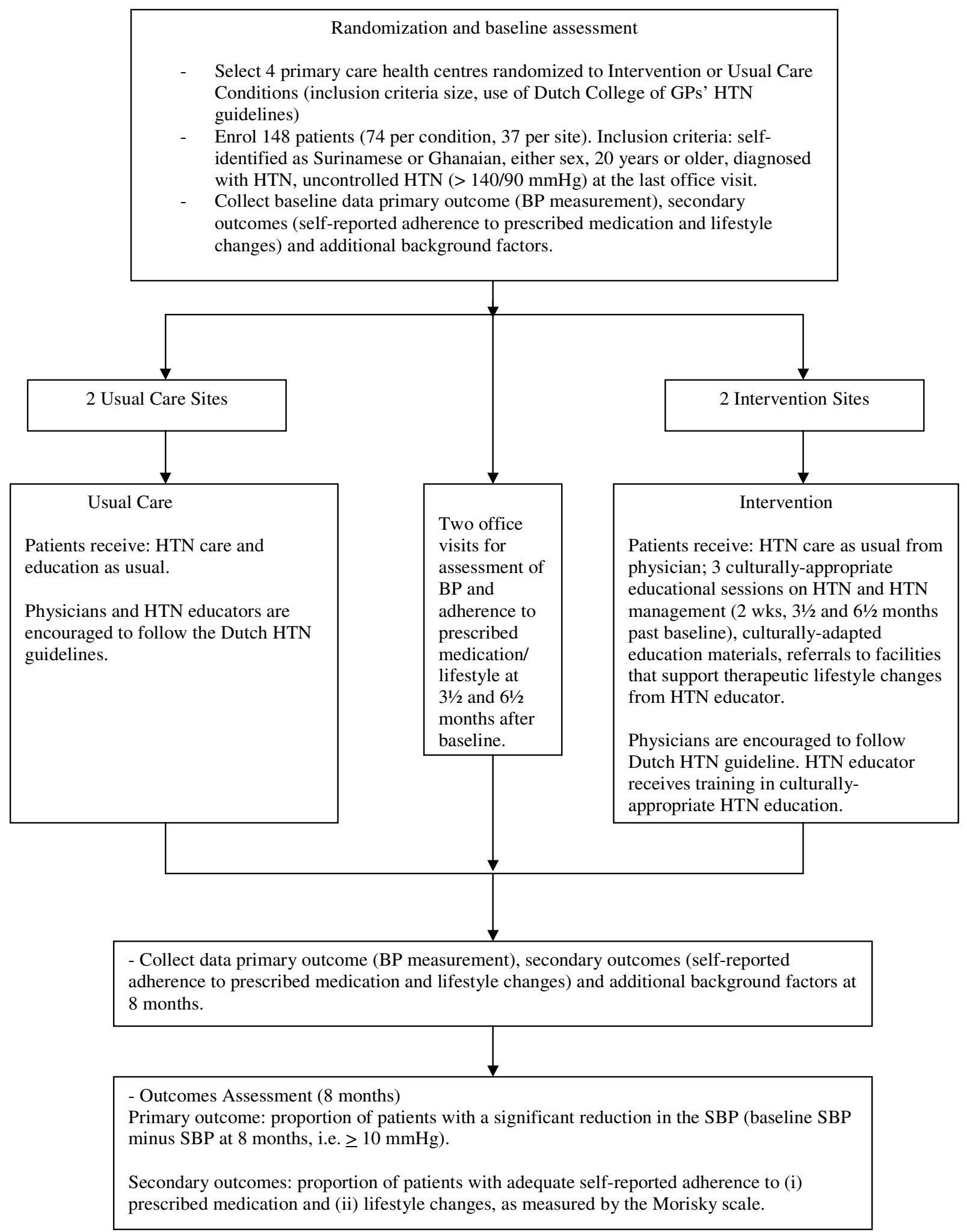

Figure I Study design. 
all patients who meet the inclusion criteria for HTN, age and sex. As Dutch EMRs provide no information on patient ethnicity, attending physicians will help to identify Ghanaian and Surinamese patients according to their knowledge of the patients or their names. The relevant patients' GPs will be informed of their potential eligibility, and the GPs will be asked for their permission to enrol the patients in the study. Eligible patients will receive a letter from the researchers by post, which will have been cosigned by their GP. This letter, which will be written in Dutch (Surinamese patients) or English (Ghanaian patients), will inform patients about the study and invite them to participate. It will include a pre-paid postcard that can be sent back to the research staff if the patient concerned wishes to participate in the study. If a patient fails to react, a member of the research team will call him or her to offer more information about the study and discuss participation.

If a patient expresses interest in participating, a staff member will check whether they meet the eligibility criteria, and will ask whether they are willing to sign an informed consent form. Eligible patients who agree to sign this form will be invited to attend a one-hour baseline assessment meeting. After making sure that the patient has signed the consent form, a research assistant (RA) will use this meeting to assess a patient's SBP and DBP, height, weight, and BMI, using standardized techniques (see below), and to obtain self-reporting measures using the baseline questionnaire. Patients who do not meet the inclusion criteria for BP or ethnicity will not be included in the study. These patients will instead receive the standard five minutes of coaching about HTN care from the RA who conducts the baseline measurement. Patients from IC practices will be assigned to the IC condition, while those from UC practices will be assigned to the UC condition. The RA, who will be blinded for the IC of the patient's PCHC, will refer all eligible patients to their PCHC's GP assistant. The GP assistant will then provide patients with a letter outlining further steps in the study. They will also make appointments with patients for follow-up office visits, in line with instructions received from the research staff. To encourage participation, all patients who are included in the study will be promised a small reward or present (value of 25 euros) after they have completed the final assessments, regardless of the intervention site.

\section{Interventions}

Intervention condition (IC)

Patients in the IC practices will receive HTN care as recommended in the Dutch clinical guidelines. Instead of the standard counselling to facilitate adherence to medication and therapeutic lifestyle changes, patients will receive: (i) three culturally-appropriate HTN education (CAHE) sessions over a period of six and a half months, conducted by a trained NP; (ii) culturally-specific, educational written material; and (iii) if necessary, referrals to neighbourhood facilities that may help Surinamese and Ghanaian patients to adopt healthier lifestyles. Moreover, (iv) prior to the second and third counselling session, an assessment of each patient's BP and self-reported medication and lifestyle adherence will be made using standardized measures. These measures are described below.

\section{Culturally-appropriate HTN education (CAHE)}

The first session will take place two weeks after the baseline assessment interview, and the next two sessions will occur three and six months thereafter. The Dutch clinical guidelines recommend the 5 As [19] as the preferred strategy for supporting patients in achieving treatment goals, such as adherence to prescribed medication, dietary changes, weight loss, reduced sodium intake, increased physical activity and moderate use of alcohol [1]. While CAHE also uses this framework, it has the additional aim of eliciting and discussing culturally-specific aspects of patients' perceptions of HTN and HTN treatment. This method is based on the work of Arthur Kleinman [33], as well as more recent approaches to improving adherence in patients with HTN [34] that were further adapted and piloted in our previous study. In short, after identifying potential communication barriers and establishing a rapport with the patient, the first session will focus on the patient's beliefs about HTN. The next two sessions will deal with the daily challenges presented by achieving HTN treatment goals within the broader context of the patient's life (see Additional file 1 and 2).

\section{Provision of information}

Patients will also be given information leaflets that provide answers to frequently-asked questions about HTN. These will be designed to address the specific languages, customs, habits, norms and dietary cultures that characterize the Surinamese and Ghanaian communities.

\section{Supporting healthier lifestyles}

If necessary, patients will be referred to neighbourhood facilities offering healthier lifestyle support that is tailored to Surinamese and Ghanaian patients, based on a referral list that will be established for this purpose.

\section{The counsellor}

In order to ensure treatment fidelity and to avoid previously identified organizational- and healthcare-related obstacles to implementation, we will appoint one NP who will provide CAHE to all IC patients. The NP will have extensive training and experience in using the counselling approach recommended in the Dutch clinical guidelines (the $5 \mathrm{As}$ ). Prior to the intervention, she will receive additional training in order to improve her knowledge of HTN in the Ghanaian and Surinamese communi- 
ties, general cross-cultural counselling techniques, and the specific CAHE method that will be used in this study. The training manual and methods that were developed during our previous study will be used for this purpose. During the trial, the NP will also be offered an opportunity to receive feedback from a member of the research team.

\section{UC condition}

Patients in UC sites will receive standard HTN care and education, following the recommendations in the Dutch clinical guidelines [1]. After completing the baseline assessment, each patient will be given appointments for two office visits at three-and-a-half and six-and-a-half months thereafter for the assessment of their BP and selfreported adherence to medication and lifestyle changes.

Regardless of the randomization condition, it will be possible to charge all office visits carried out in the context of this trial to health insurance companies.

\section{Outcomes, measures and data analysis}

\section{Primary outcome}

The primary outcome measure is the proportion of patients with a significant reduction in the SBP (10 $\mathrm{mmHg}$; $\mathrm{SD}=15$ ) at eight months after inclusion.

\section{Secondary outcomes}

The secondary outcome measures are: (i) the proportion of patients with adequate adherence to prescribed medication at eight months after inclusion; and (ii) the proportion of patients with adequate adherence to lifestyle recommendations at eight months after inclusion.

Data will also be collected with respect to factors that characterize the patient group (baseline demographics, baseline medical chart data) and a number of factors that may influence patients' HTN management (perceptions of HTN, perceptions of medications, self-efficacy, experience of social support in HTN management, and level of satisfaction with care). In addition, data relating to the process of implementing the intervention will be collected.

\section{Measures}

The measurements to be studied can be divided into five categories: (1) physiological measures, (2) self-reporting measures, (3) pharmacy data, (4) chart data and (5) process data. Table 1 summarizes the measures according to the timeline. A trained RA will conduct all physiological and self-reporting measures at baseline and at eight months. Attending NPs at the IC and UC practices will take a subsection of these measures (BP and self-reported adherence to medication and life style changes) at three-

Table I: Measures used in the trial and timeline

\begin{tabular}{|c|c|c|c|c|c|c|}
\hline Measures & Baseline & $\begin{array}{c}2 \\
\text { wks }\end{array}$ & $\begin{array}{c}3 \mathrm{I} / 2 \\
\text { mnths }\end{array}$ & $\begin{array}{c}6 \mathrm{I} / 2 \\
\text { mnths }\end{array}$ & $\begin{array}{c}8 \\
\text { mnths }\end{array}$ & $\begin{array}{c}9 \\
\text { mnths }\end{array}$ \\
\hline \multicolumn{7}{|l|}{ Physiological measures } \\
\hline - Office BP measurements & $\mathrm{X}$ & & $\mathrm{X}$ & $\mathrm{X}$ & $\mathrm{x}$ & \\
\hline - Height, weight, BMI & $\mathrm{X}$ & & & & $\mathrm{x}$ & \\
\hline - Hip and waist size & $\mathrm{x}$ & & & & $x$ & \\
\hline \multicolumn{7}{|l|}{ Self-reporting measures } \\
\hline - Patient demographics & $\mathrm{x}$ & & & & & \\
\hline - Additional cardiovascular risk factors (physical activity, smoking, alcohol, sodium intake) & $\mathrm{X}$ & & & & $\mathrm{X}$ & \\
\hline - Medication adherence & $\mathrm{X}$ & & $\mathrm{X}$ & $\mathrm{X}$ & $\mathrm{X}$ & \\
\hline - Adherence to lifestyle recommendations & $\mathrm{X}$ & & $\mathrm{X}$ & $\mathrm{X}$ & $x$ & \\
\hline - Perceptions of HTN & $x$ & & & & $x$ & \\
\hline - Perceptions of medication & $\mathrm{x}$ & & & & $x$ & \\
\hline - Self efficacy & $\mathrm{x}$ & & & & $x$ & \\
\hline - Experience of social support in HTN management & $\mathrm{x}$ & & & & $\mathrm{x}$ & \\
\hline - Satisfaction with care & $\mathrm{x}$ & & & & $\mathrm{X}$ & \\
\hline - Discrimination & $\mathrm{x}$ & & & & $x$ & \\
\hline - Perceived stress & $x$ & & & & $x$ & \\
\hline \multicolumn{7}{|l|}{ Pharmacy data } \\
\hline - Refill dates & $\mathrm{x}$ & & $\mathrm{x}$ & $\mathrm{x}$ & $\mathrm{x}$ & \\
\hline \multicolumn{7}{|l|}{ Chart data } \\
\hline - Prescribed medication & $\mathrm{x}$ & & & & & \\
\hline - Prescribed lifestyle measures & $\mathrm{x}$ & & & & & \\
\hline - Co-morbidity & $x$ & & & & & \\
\hline \multicolumn{7}{|l|}{ Process data } \\
\hline - Registration office visits, patient drop-out & $x$ & $x$ & $\mathrm{x}$ & $x$ & $x$ & \\
\hline - Patients interviews & & & & & & $\mathrm{x}$ \\
\hline
\end{tabular}


and-a-half and at six-and-a-half months. A member of the research staff will collect pharmacy and chart data. Time registration and patient drop-out data for the process analysis will be collected using registrations made by the RA and the NPs. A member of the research staff will conduct post-intervention interviews with patients.

\section{Physiological measures}

$\mathrm{BP}$ will be measured three times using an automated BP monitor (Omron 705-IT), with the patient seated comfortably for five minutes. The average of the last two readings will be used as a measure for each visit. The same procedure will be used by trained NPs during office visits at three-and-a-half and six-and-a-half months after baseline.

Height and weight will be measured in the absence of shoes and heavy clothing, using a tape rule and a validated scale respectively. All measurements will be recorded to the nearest $0.1 \mathrm{~cm}$ and $0.1 \mathrm{~kg}$. These measures will be used to compute a patient's BMI. Waist size will be measured by putting the tape horizontally around the smallest part of the waist. Hip size will be measured by putting the tape horizontally around the widest part of the hips.

\section{Self-reporting measures}

In order to collect socio-demographic data describing the study population, we have adapted an instrument used in an earlier study [35]. The variables will include age, gender, self-identified ethnicity, educational level, employment status, household income, marital status, household composition, duration of stay in the Netherlands, duration of HTN, and health insurance status.

To establish the presence of additional cardiovascular risk factors, we will use an instrument that contains six questions relating to physical activity, smoking, alcohol and sodium intake. Questions will be based on an instrument used in a previous study [35].

Adherence to prescribed antihypertensive medication will be measured with the widely-used four-item scale developed by Morisky [36]. This instrument has been well validated in studies of African American inner-city populations [37-39]. The scale asks patients to respond with "yes" or "no" to a set of four questions. A positive response to any question indicates a problem with adherence. Each positive answer is assigned a score of 1 . The total possible score is 4 , with a higher score indicating a poorer level of adherence. In this study, patients with a score of $\geq 1$ will be categorized as non-adherent. In addition, six items will be used that are based on our previous study [40].
To assess adherence to lifestyle recommendations, we will use four items derived from the Morisky scale [36] adapted for the purpose of this study.

Perceptions of HTN and HTN treatment will be measured using 52 items: of these, nine will be from the IPQ-brief [41], 18 will be from the IPQ-R HTN [42], five will be derived from our earlier studies on perceptions of HTN and HTN management among Surinamese and Ghanaians $[40,43,44]$, and 20 items will be adapted from the IPQ-R [45]. The validity and the reliability of the IPQ-brief and the IPQ-R are well established $[41,45,46]$. Patients' perceptions of antihypertensive medications will be measured using the ten-item BMQ [47], an instrument that has good internal consistency and validity $[48,49]$. In addition to this, we will add three items that are based on our previous study [40].

To measure patients' self-efficacy in HTN management, the 13-item MASES-R will be used. This instrument has good internal consistency and predictive and convergent validity [50].

Social support will be measured using the 12-item DUSOCS [51], which, as demonstrated by previous studies, has good validity and reliability [37].

Satisfaction with care will be measured using six items of the Consumer Quality Index-diabetes [52]. Similar Consumer Quality Index instruments have demonstrated good construct and discriminating validity and internal consistency in previous Dutch studies $[53,54]$. In addition, five items from the Quote-Migrant will be used, which has been specifically developed to measure the experienced quality of care in migrant populations [55].

Socio-demographic data will only be measured at baseline. Most of the other self-reporting measures will be collected at baseline and at eight months thereafter.

The Morisky scale will also be used to assess adherence to medication and lifestyle recommendations during the second and third office visits, respectively three-and-a-half and six-and-a-half months after the baseline assessment. Patients will be asked to complete these questionnaires in practice waiting rooms, prior to meeting the NP.

Discrimination and stress will be measured with two scales from the CAATCH trial [56].

\section{Pharmacy data}

In addition to self-reporting data, pharmacy data will be used to check self-reported adherence to medication. These data will allow us to measure refill adherence; that is, the interval between the day when a prescription ends, 
and the day on which a new prescription is refilled [57]. A patient is defined as adherent if the prescription is refilled within seven days of the designated refill date. This method has also been used in other studies [58].

\section{Chart data}

In addition to self-reported biographical and disease data, relevant chart data from the patients' charts will be extracted from the EMR as soon as patients have been included in the study. The following data will be extracted: SBP and DBP measured during the last two office visits; prescribed medication; the presence of additional risk factors for cardiovascular disease (physical exercise, diet, sodium, smoking, alcohol, height, weight, BMI, waist and hip circumference, glucose, cholesterol); and the presence of previous cardiovascular diseases (myocardial infarction, decompenstatio cordis, TIA, CVA), kidney insufficiency, liver disease, dementia or mental health problems. In a previous study, we found that GP registration data can sometimes be incomplete [40]. If these data are indeed sufficient, they will be used as additional information to describe the background characteristics of the study population, and to control for confounders.

\section{Process data}

Time registrations for office visits and additional activities made by the RA and NPs will be used to make a cost analysis of the intervention. Registration data on patient dropout will be used to analyse the feasibility of undertaking the intervention for the patients groups in question. Moreover, qualitative interviews with patients in the IC and the UC conditions are planned to explore patients' experiences of barriers to and incentives for participating in the study. We will also enquire about non-reimbursable expenses that might arise from participating in the study, such as travel costs and costs related to lifestyle changes.

\section{Blinding}

Due to the nature of the interventions concerned, complete blinding of investigators and professionals working in the PCHCs would be impossible. However, the RA who will conduct the assessments at baseline and at eight months will be masked for the IC of the PCHCs. All BP measurements will be taken with a standard automated measurement device that can prevent ascertainment bias. Even though physicians may be aware of the IC of their healthcare centre, we consider contamination to be unlikely on the grounds that the intervention (CAHE) will require the use of specific expertise and tools. Finally, data managers will be strictly blinded for the IC. All preliminary data will be stored in one dataset until the end of the intervention.

\section{Planned data analysis}

All imported data will be controlled at random for miscodings. As a first step, univariate analyses will be performed to compare the distribution of variables and to identify abnormalities.

Primary and secondary outcomes will be calculated according to the "intention to treat" principle. The primary outcome measure, which will be calculated for every patient, will be the observed baseline SBP minus the SBP at eight months. Subsequently, the mean within group difference will be calculated separately for patients in the IC and UC conditions. Finally, the difference between these within group differences will be calculated with the accompanying SD. As stated previously, we regard a reduction of the SBP of $10 \mathrm{mmHg}$ with a SD of 15 and a two-sided alpha of 5\% in the IC group (as compared to the UC group) as a clinically relevant difference.

The secondary outcomes will be calculated using the dichotomous outcomes of measurements on the Morisky scale with respect to adherence to prescribed medication and lifestyle changes. The analytical procedure will be similar to that described for the primary outcome measure.

In a further analysis of the primary and secondary outcomes, we will use univariate and multi-level analyses that will include other data that may potentially affect the outcomes. If the $\mathrm{N}$ is sufficient, we will perform separate subgroup analyses for gender and ethnicity. A p of 0.05 will be used as the critical value for all analyses. For subgroup analyses, the p value will be corrected according to the number of analyses conducted.

\section{Discussion}

In western countries, there is an urgent need to improve BP control among hypertensive patients of African descent. In the US, trials of interventions to enhance BP control and patient adherence to prescribed medications and lifestyle changes have focused on African American patients. Two of these trials are currently taking place $[11,56]$ and one recently finished [37]. To our knowledge, the present study is one of the first European trials to address this issue. The US trials are evaluating the effect of interventions that are based on the principle of motivational interviewing [59]. The specific contribution of the present study is that the intervention builds on the principles of "culturally-appropriate counselling" [60], which can be used to supplement motivational interviewing. Another key characteristic of the intervention is that it has been developed and piloted using data from patients' own perceptions of HTN $[40,43,44]$. Moreover, drawing on theories of effective quality improvement in primary care 
$[30,31]$, the feasibility for implementation by care providers has already been established in a previous pilot study.

If the results are positive, we hope that this intervention will find wider application, so that it may contribute to efforts to reduce ethnic disparities in HTN outcomes.

\section{Abbreviations}

(AMC): Academic Medical Center; (CAHE): Culturallyappropriate hypertension education; (DBP): Diastolic blood pressure; (EMR): Electronic medical record; (GP): General practitioner; (GP assistant): General practice assistant; (HTN): Hypertension; (IC): Intervention condition; (NP): Nurse practitioner; (PCHC): Primary care health center; (RA): Research assistant; (SD): Standard deviation; (SBP): Systolic blood pressure; (UP2): Under Pressure 2; (UC): Usual care condition; 5 As: (ask, advise, assess, assist, arrange).

\section{Competing interests}

The authors declare that they have no competing interests.

\section{Authors' contributions}

$\mathrm{JH}$ wrote the research proposal for the funding organization, and drafted the manuscript of this paper. EB made a major contribution to the design of the research proposal, prepared the study protocol for ethical approval together with $\mathrm{JH}$, and helped to draft this paper. CA participated in the design of the research proposal and the final drafts of the study protocol. EM commented on the final draft of the study protocol. All four authors read and approved the final manuscript of the paper.

\section{Additional material}

\section{Additional file 1}

Summary of three culturally-appropriate hypertension education sessions. The information provided describes the content of culturally-appropriate hypertension education.

Click here for file

[http://www.biomedcentral.com/content/supplementary/14726963-9-193-S1.DOC]

\section{Additional file 2}

Topic list for eliciting a patient's explanatory model of hypertension. The information provided describes the content of culturally-appropriate hypertension education.

Click here for file

[http://www.biomedcentral.com/content/supplementary/14726963-9-193-S2.DOC]

\section{Acknowledgements}

The authors wish to thank Professor Patrick Bindels and Professor Karien Stronks for their intellectual contribution to the design of the research proposal and the protocol for this study. They also wish to thank Dr Bert-Jan van den Born, Dr Hans Grundmeijer and members of the AMC's Medical Ethics Committee for their comments on the protocol. We greatly appreciated the statistical support provided by $\mathrm{Dr}$ Robert Lindeboom, as well as the support provided by our research assistant, Corine Buers, who helped us to make the final selection of the research instruments that will be used in the trial. We also received invaluable support from Parad Keijser, in translating the protocol to practice. The Dutch Organization for Health Research Development (ZonMw) provided a grant for this study (grant no. 122000008).

\section{References}

I. Smulders YM, Burgers JS, Scheltens T, van Hout BA, Wiersma T, Simoons ML: Clinical practice guideline for cardiovascular risk management in the Netherlands. Neth J Med 2008, 66:169-174.

2. Cappuccio FP: Ethnicity and cardiovascular risk: variations in people of African ancestry and South Asian origin. J Hum Hypertens 1997, I I:571-576.

3. Raleigh VS: Diabetes and hypertension in Britain's ethnic minorities: implications for the future of renal services. $B M J$ 1997, 3 | 4:209-2|3.

4. Douglas JG, Bakris GL, Epstein M, Ferdinand KC, Ferrario C, Flack JM, Jamerson KA, Jones WE, Haywood J, Maxey R, et al.: Management of High Blood Pressure in African Americans: Consensus Statement of the Hypertension in African Americans Working Group of the International Society on Hypertension in Blacks. Arch Intern Med 2003, 163:525-54I.

5. Agyemang C, Bindraban N, Mairuhu G, Montfrans G, Koopmans R, Stronks K: Prevalence, awareness, treatment, and control of hypertension among Black Surinamese, South Asian Surinamese and White Dutch in Amsterdam, The Netherlands: the SUNSET study. J Hypertens 2005, 23:197I-1977.

6. Born BJ van den, Koopmans RP, Groeneveld JO, van Montfrans GA Ethnic disparities in the incidence, presentation and complications of malignant hypertension. J Hypertens 2006, 24:2299-2304.

7. Agyemang C, Bruijnzeels MA, Owusu-Dabo E: Factors associated with hypertension awareness, treatment, and control in Ghana, West Africa. J Hum Hypertension 2005, 20:67-7I.

8. Agyemang C, van VI, Koopmans R, Stronks K: Factors associated with hypertension awareness, treatment and control among ethnic groups in Amsterdam, the Netherlands: the SUNSET study. J Hum Hypertens 2006, 20:874-88I.

9. Bosworth HB, Dudley T, Olsen MK, Voils Cl, Powers B, Goldstein MK, Oddone EZ: Racial Differences in Blood Pressure Control: Potential Explanatory Factors. The American Journal of Medicine 2006, II 9:70.

10. Miller NH, Hill M, Kottke T, Ockene IS: The multilevel compliance challenge: recommendations for a call to action. A statement for healthcare professionals. Circulation 1997, 95: $1085-1090$.

II. Cooper L, Roter D, Bone L, Larson S, Miller E, Barr M, Carson K, Levine $D$ : A randomized controlled trial of interventions to enhance patient-physician partnership, patient adherence and high blood pressure control among ethnic minorities and poor persons: study protocol NCT00 123045 . Implementation Science 2009, 4:7.

12. De Backer G, Ambrosioni E, Borch-johnsen K, Brotons C, Cifkova R, Dallongeville J, Ebrahim S, Faergeman O, Graham I, Mancia G, et al:: European guidelines on cardiovascular disease prevention in clinical practice: Third Joint Task Force of European and other Societies on Cardiovascular Disease Prevention in Clinical Practice (constituted by representatives of eight societies and by invited experts). Eur Heart J 2003, 24:160I-1610.

13. Wiersma T, Walma EP, Thomas S, Assendelft W]: [Summary of the practice guideline 'Hypertension' (third division) from the Dutch College of General Practitioners]. Ned Tijdschr Geneeskd 2004, | 48:923-93|.

14. Kleinman A, Eisenberg L, Good B: Culture, Illness, and Care: Clinical Lessons From Anthropologic and Cross-Cultural Research. Focus 2006, 4:140-149.

15. Boulware LE, Daumit GL, Frick KD, Minkovitz CS, Lawrence RS, Powe NR: An evidence-based review of patient-centered 
behavioral interventions for hypertension. American Journal of Preventive Medicine 200I, 21:22I-232.

16. Chobanian AV, Bakris GL, Black HR, Cushman WC, Green LA, Izzo JL Jr, Jones DW, Materson BJ, Oparil S, Wright JT Jr, et al:: The Seventh Report of the Joint National Committee on Prevention, Detection, Evaluation, and Treatment of High Blood Pressure: The JNC 7 Report. JAMA 2003, 289:2560-257I.

17. Krousel-Wood M, Hyre A, Muntner P, Morisky D: Methods to improve medication adherence in patients with hypertension: current status and future directions. Curr Opin Cardiol 2005, 20:296-300.

18. Saha S: Patient Centeredness, Cultural Competence and Healthcare Quality. Journal of the National Medical Association 2008, 100:1275-1284.

19. Whitlock EP, Orleans CT, Pender N, Allan J: Evaluating primary care behavioral counseling interventions: An evidence-based approach. American Journal of Preventive Medicine 2002, 22:267-284.

20. Heurtin-Roberts $S$, Reisin E: The relation of culturally influenced lay models of hypertension to compliance with treatment. Am J Hypertens 1992, 5:787-792.

21. Morgan M: The significance of ethnicity for health promotion: patients' use of anti-hypertensive drugs in inner London. Int J Epidemiol 1995, 24(Suppl I):S79-S84

22. Brown CM, Segal R: The effects of health and treatment perceptions on the use of prescribed medication and home remedies among African American and white American hypertensives. Soc Sci Med 1996, 43:903-917.

23. Brown CM, Segal R: Ethnic differences in temporal orientation and its implications for hypertension management. J Health Soc Behav 1996, 37:350-36I.

24. Wilson RP, Freeman A, Kazda MJ, Andrews TC, Berry L, Vaeth PAC, Victor RG: Lay beliefs about high blood pressure in a low- to middle-income urban African-American community: an opportunity for improving hypertension control. The American Journal of Medicine 2002, I I 2:26-30.

25. Helman CG: Culture, health and illness London: Hodder Arnold; 2007.

26. Middelkoop BJC, Geelhoed-Duijvestijn PHLM, Wal G van der: Effectiveness of Culture-Specific Diabetes Care for Surinam South Asian Patients in the Hague: A randomized controlled trial/controlled before-and-after study. Diabetes Care 200I, 24:1997-1998.

27. Hawthorne K, Robles Y, Cannings-John R, Edwards AG: Culturally appropriate health education for type 2 diabetes mellitus in ethnic minority groups. Cochrane Database Syst Rev 2008:CD006424.

28. Beach MC, Gary TL, Price EG, Robinson K, Gozu A, Palacio A, Smarth C, Jenckes M, Feuerstein C, Bass EB, et al:: Improving health care quality for racial/ethnic minorities: a systematic review of the best evidence regarding provider and organization interventions. BMC Public Health 2006, 6: 104.

29. Grimshaw JM, Shirran L, Thomas R, Mowatt G, Fraser C, Bero L, Grilli $R$, Harvey E, Oxman A, O'Brien MA: Changing provider behavior: an overview of systematic reviews of interventions. Med Care 200I, 39:112-45.

30. Grol R, Grimshaw J: From best evidence to best practice: effective implementation of change in patients' care. Lancet 2003 , 362: $1225-1230$

31. Grol R: Changing physicians' competence and performance: finding the balance between the individual and the organization. J Contin Educ Health Prof 2002, 22:244-25I.

32. Whelton PK, He J, Appel LJ, Cutler JA, Havas S, Kotchen TA, Roccella EJ, Stout R, Vallbona C, Winston MC, et al.: Primary prevention of hypertension: clinical and public health advisory from The National High Blood Pressure Education Program. JAMA 2002, 288: $1882-1888$

33. Kleinman A, Eisenberg L, Good B: Culture, illness, and care: clinical lessons from anthropologic and cross-cultural research. Ann Intern Med 1978, 88:25I-258.

34. Betancourt JR, Carrillo JE, Green AR: Hypertension in multicultural and minority populations: linking communication to compliance. Curr Hypertens Rep 1999, I:482-488.

35. Bindraban NR: The Cardiovascular Risk Profile of Hindustani and Creole Surinamese in the Netherlands compared to white Dutch people. In PhD Thesis AMC-University of Amsterdam; 2006.
36. Morisky DE, Green LW, Levine DM: Concurrent and predictive validity of a self-reported measure of medication adherence. Med Care 1986, 24:67-74.

37. Ogedegbe G, Schoenthaler A, Richardson T, Lewis L, Belue R, Espinosa E, Spencer J, Allegrante JP, Charlson ME: An RCT of the effect of motivational interviewing on medication adherence in hypertensive African Americans: rationale and design. Contemp Clin Trials 2007, 28:169-181.

38. Shea S, Misra D, Ehrlich MH, Field L, Francis CK: Correlates of nonadherence to hypertension treatment in an inner-city minority population. Am J Public Health 1992, 82:1607-16I2.

39. Schoenthaler A, Chaplin WF, Allegrante JP, Fernandez S, Diaz-Gloster $M$, Tobin JN, Ogedegbe G: Provider communication effects medication adherence in hypertensive African Americans. Patient Educ Couns 2009, 75: 185-191.

40. Beune EJ, Haafkens JA, Agyemang C, Schuster JS, Willems DL: How Ghanaian, African-Surinamese and Dutch patients perceive and manage antihypertensive drug treatment: a qualitative study. J Hypertens 2008, 26:648-656.

4I. Broadbent E, Petrie KJ, Main J, Weinman J: The brief illness perception questionnaire. J Psychosom Res 2006, 60:63I-637.

42. Clatworthy J, Horne R, Buick D, Weinman J: your views about your high blood pressure. [http://www.uib.no/ipa/pdf/IPQ Hypertension-New.pdf]. 2009-09-06

43. Beune EJAJ, Haafkens JA, Schuster JS, Bindels PJE: 'Under pressure': how Ghanaian, African-Surinamese and Dutch patients explain hypertension. J Hum Hypertens 2006, 20:946-955.

44. Beune EJ, Haafkens JA, Agyemang C, Bindels PJ: Inhibitors and enablers of physical activity in multiethnic hypertensive patients: qualitative study. J Hum Hypertens 2009 in press.

45. Moss-Morris R, Weinman J, Petrie K, Horne R, Cameron L, Buick D: The Revised Illness Perception Questionnaire (IPQ-R). Psychology and Health 2002, 17:1-16.

46. Figueiras MJ, Alves NC, Marcelino D, Cortes MA, Weinman J, Horne R: Assessing lay beliefs about generic medicines: Development of the generic medicines scale. Psychol Health Med 2009, 14:3II-32I.

47. Horne R, Weinman J, Hankins M: The beliefs about medicines questionnaire: The development and evaluation of a new method for assessing the cognitive representation of medication. Psychology \& Health 1999, I 4: I-24.

48. Theunissen NC, de Ridder DT, Bensing JM, Rutten GE: Manipulation of patient-provider interaction: discussing illness representations or action plans concerning adherence. Patient Educ Couns 2003, $51: 247-258$.

49. Ross S, Walker A, MacLeod MJ: Patient compliance in hypertension: role of illness perceptions and treatment beliefs. J Hum Hypertens 2004, 18:607-613.

50. Fernandez S, Chaplin W, Schoenthaler AM, Ogedegbe G: Revision and validation of the medication adherence self-efficacy scale (MASES) in hypertensive African Americans. I Behav Med 2008, 31:453-462.

5I. Parkerson GR Jr, Broadhead WE, Tse CK: Validation of the Duke Social Support and Stress Scale. Fam Med I99I, 23:357-360.

52. Consumer Quality Index-diabetes Utrecht, Nivel [http://
[ www nivel nl/oc2/page asp'pageid=8266]. 2009-09-06

53. Stubbe J, Gelsema T, Delnoij D: The Consumer Quality Index Hip Knee Questionnaire measuring patients' experiences with quality of care after a total hip or knee arthroplasty. BMC Health Services Research 2007, 7:60.

54. Stubbe JH, Brouwer W, Delnoij DMJ: Patients' experiences with quality of hospital care: the Consumer Quality Index Cataract Questionnaire. BMC Ophthalmology 2007, 7:14.

55. Harmsen JAM, Bernsen RMD, Bruijnzeels MA, Meeuwesen L: Patients' evaluation of quality of care in general practice: What are the cultural and linguistic barriers? Patient Education and Counseling 2008, 72:155-162.

56. Ogedegbe G, Tobin JN, Fernandez S, Gerin W, Diaz-Gloster M, Cassells A, Khalida C, Pickering T, Schoenthaler A, Ravenell J: Counseling African Americans to Control Hypertension (CAATCH) Trial: A Multi-Level Intervention to Improve Blood Pressure Control in Hypertensive Blacks. Circ Cardiovasc Qual Outcomes 2009, 2:249-256.

57. Wetzels GE, Nelemans PJ, Schouten JS, Dirksen CD, Weijden T van der, Stoffers HE, Janknegt R, de Leeuw PW, Prins MH: Electronic monitoring of adherence as a tool to improve blood pressure 
control. A randomized controlled trial. Am J Hypertens 2007, 20:119-125.

58. Willey C, Redding C, Stafford J, Garfield F, Geletko S, Flanigan T, Melbourne K, Mitty J, Caro J]: Stages of change for adherence with medication regimens for chronic disease: development and validation of a measure. Clin Ther 2000, 22:858-87I.

59. Britt E, Hudson SM, Blampied NM: Motivational interviewing in health settings: a review. Patient Education and Counseling 2004, 53:147-155.

60. Saha S, Beach MC, Cooper LA: Patient centeredness, cultural competence and healthcare quality. J Natl Med Assoc 2008, 100:1275-1285.

\section{Pre-publication history}

The pre-publication history for this paper can be accessed here:

http://www.biomedcentral.com/1472-6963/9/193/pre

pub

Publish with Bio Med Central and every scientist can read your work free of charge

"BioMed Central will be the most significant development for disseminating the results of biomedical research in our lifetime. "

Sir Paul Nurse, Cancer Research UK

Your research papers will be:

- available free of charge to the entire biomedical community

- peer reviewed and published immediately upon acceptance

- cited in PubMed and archived on PubMed Central

- yours - you keep the copyright

Submit your manuscript here:

http://www.biomedcentral.com/info/publishing_adv.asp 